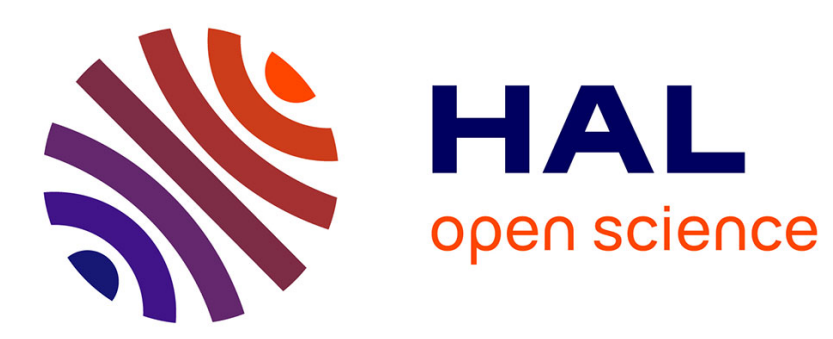

\title{
Linear Sampling and Magnification Technique Based on Phase Modulators and Dispersive Elements: the Temporal Lenticular Lens
}

\author{
Javier Nuño, Christophe Finot, Julien Fatome
}

\section{- To cite this version:}

Javier Nuño, Christophe Finot, Julien Fatome. Linear Sampling and Magnification Technique Based on Phase Modulators and Dispersive Elements: the Temporal Lenticular Lens. Optical Fiber Technology, 2017, 36, pp.125-129. 10.1016/j.yofte.2017.03.006 . hal-01389069

\section{HAL Id: hal-01389069 \\ https://hal.science/hal-01389069}

Submitted on 27 Oct 2016

HAL is a multi-disciplinary open access archive for the deposit and dissemination of scientific research documents, whether they are published or not. The documents may come from teaching and research institutions in France or abroad, or from public or private research centers.
L'archive ouverte pluridisciplinaire HAL, est destinée au dépôt et à la diffusion de documents scientifiques de niveau recherche, publiés ou non, émanant des établissements d'enseignement et de recherche français ou étrangers, des laboratoires publics ou privés. 


\title{
Linear Sampling and Magnification Technique Based on Phase Modulators and Dispersive Elements: the Temporal Lenticular Lens
}

Javier Nuño*, Christophe Finot, Julien Fatome

Laboratoire Interdisciplinaire Carnot de Bourgogne, UMR 6303 CNRS-Université Bourgogne Franche-Comté, 9 av. A. Savary, 21078 Dijon Cedex, France

\author{
${ }^{*}$ Corresponding author: \\ E-mail address:j.nugno@gmail.com
}

\begin{abstract}
In this work, we exploit the space/time duality in optics to implement a temporal lenticular lens allowing to simultaneously sample and magnify an arbitrary-shaped optical signal. More specifically, by applying a sinusoidal phase-modulation, the signal under test is propagated through a discrete dispersive element that samples and magnifies its initial waveform. Thanks to this temporal lenticular lens, optical sampling associated to a magnification factor of 3.6 is experimentally demonstrated at a repetition rate of $10 \mathrm{GHz}$.
\end{abstract}

Keywords: All-optical signal processing; fiber devices; linear sampling; phase modulation; dispersion. 


\section{Introduction}

In modern photonic systems, the sampling process has widespread applications in the fields of optical communications, metrology, clocking, sensing, spectral comb or arbitrary waveform generation. In this context, nonlinear effects have been demonstrated as potential key technologies to develop all-optical sampling devices [1]-[9]. Most of these methods are based on the nonlinear interaction among a train of ultrashort high-power pulses and the original signal, thus requiring an external mode locked picosecond pulse source. The basic physical phenomena involved in these interactions include four-wave mixing [5], cross-phase modulation (XPM) [6], nonlinear polarization rotation [8] or Raman soliton self-frequency shift [9].

More recently, an alternative nonlinear approach has been experimentally demonstrated at a sampling rate of $40 \mathrm{GHz}$, whilst providing a simultaneous 8-dB magnification factor of the waveform [10]. Basically, during its co-propagation in a km-long fiber with a high-power sinusoidal pump beam, the waveform under test experiences a strong temporal phase modulation due to the XPM coupling. Subsequently, the signal undergoes a simultaneous temporal compression due to the normal dispersion regime of the fiber, leading to a periodic and localized nonlinear focusing effect [11]. In this new contribution, we propose a linear implementation of this process that does not require any external optical sampling beam. More precisely, here an electro-optic modulator is used to imprint the initial temporal phase modulation, while a programmable and compact spectral phase shaper enables an optimum sampling process through the formation of periodic pulses proportional to the initial waveform. Based on the analogy that can be drawn between usual spatial optics and ultrafast optics [12]-[13], such approach mimics in the temporal domain the behavior of a periodic 
lenticular lens that focuses different parts of an illuminating plane wave at equally spaced positions.

Our manuscript is organized as follows. In the second section, we describe the principle of our method and in particular, we highlight the analogy with the lenticular lens. Subsequently, we also present some design guidelines related to the magnification factor and optimum dispersion. In sections 3 , we then describe the experimental linear setup we implemented. The experimental results are presented in section 4 and are compared with numerical simulations, demonstrating an excellent agreement. Finally, in section 5, we summarize our work and trace out some conclusions and outlooks.

\section{Principle of operation and design rules}

Frequency chirp compensation of a parabolic time-dependent phase modulated signal passing through a dispersive medium imposing a quadratic spectral phase is a widely spread technique to produce ultrashort structures [14]. More generally, this focusing process can be viewed as the temporal analogue of an optical lens by using the space-time duality [15]-[16].

To imprint such an initial modulation within an incoming signal, taking advantage of the Kerr nonlinearity in optical fibers has been proved to be an attractive and efficient approach, for instance through self- or cross-phase modulation processes [17]-[19]. In a recent work, we have suggested and experimentally demonstrated an all-fibered sampling technique based on the XPM induced by a sinusoidal pump beam on the signal under test (SUT) combined to a simultaneous adiabatic compensation through normal chromatic dispersion [10]. In this 
configuration, the SUT experiences periodic local compression, leading to an efficient sampling and magnification of its waveform.

An alternative approach to imprint such initial temporal phase modulation is to benefit from electro-optic devices [20]. Based on standard off-the-shelf telecom platform, this linear method has been proved more stable, less complex as well as more power efficient than nonlinear techniques. However, lower phase modulation amplitude can be achieved, so cascading several phase modulators is often required to achieve large frequency chirps [21]. Moreover, due to experimental constraints, synthetizing a perfect temporal quadratic phase modulation is quite difficult to achieve so most of the time, the initial modulation is replaced by a sinusoidal waveform that is close to a parabola at its extrema. In this case, when driven by RF sinusoidal signals, it is well known that the phase modulation of an initial continuous wave $(\mathrm{CW})$ leads to the generation of high-repetition rate pulse trains after its propagation in a dispersive element [22]-[23]. By exploiting the space-time duality, as depicted in Fig. 1(a), such method of periodic temporal focusing presents some analogy with lenticular lenses in geometric optics where lenses are periodically reproduced and for which an incoming plane wave is focused at different equally spaced points. 

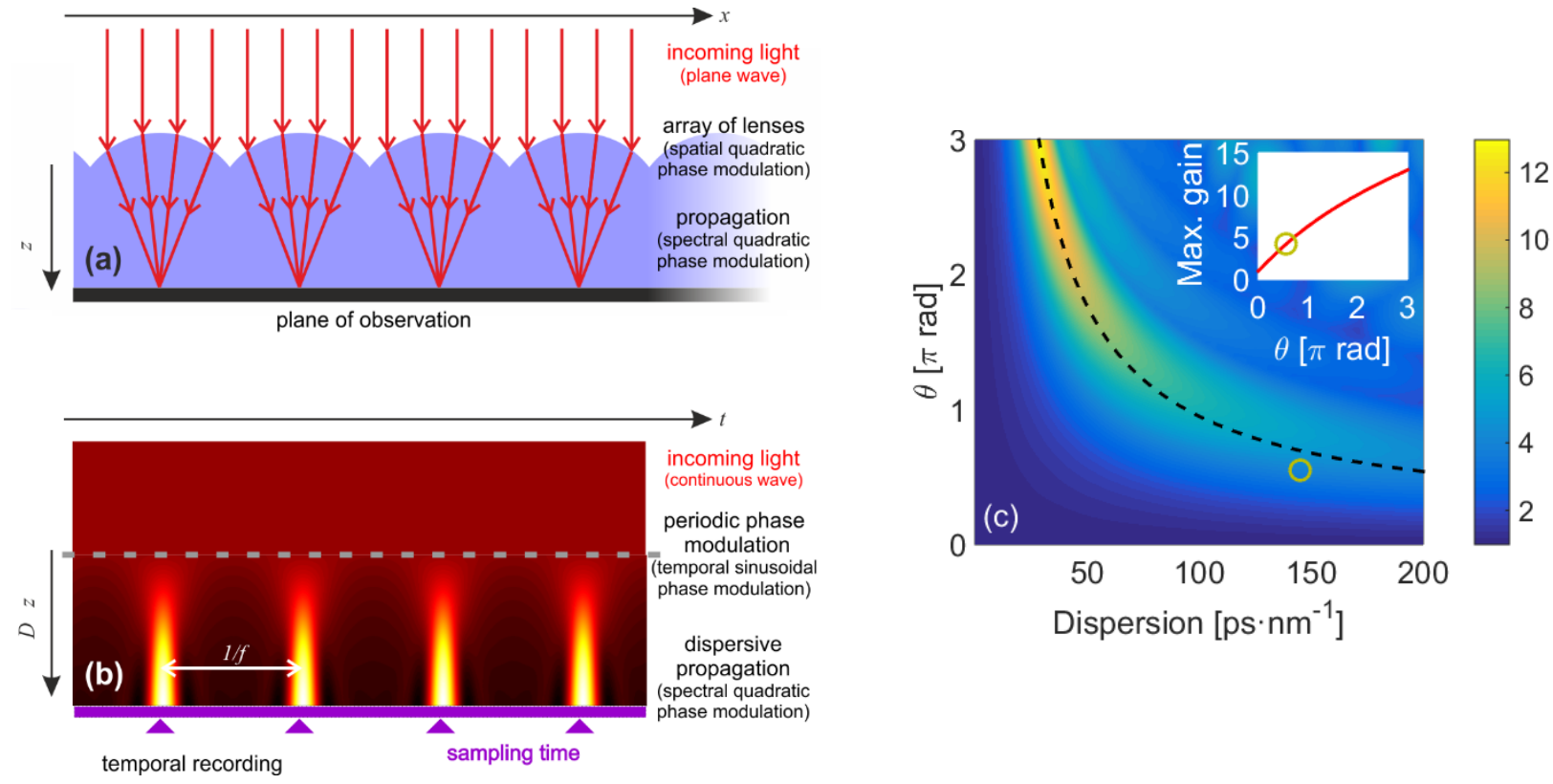

Fig. 1. (a) Lenticular lens illuminated by a plane wave. (b) Principle of the proposed temporal approach. (c) Magnifying factor as a function of both the amplitude $\theta$ of the phase modulation and the amount of chromatic dispersion. The dotted line represents the maximum magnifying factor according to the approximate prediction given by Eq. (3). In the inset, the maximum magnifying factor for each phase is plotted. The yellow circle represents the values in our experiments $\left(\theta=0.55 \pi\right.$ and $D \approx 145 \mathrm{ps} \cdot \mathrm{nm}^{-1}$ in the fiber). In both cases, the frequency is equal to $10 \mathrm{GHz}$. 
In this novel contribution, we exploit a similar physical principle as the basis of our linear sampler. As depicted in Fig. 1(b), the modulation of the temporal phase produces a frequency chirp and in a second stage, a dispersive element that can be a linear optical fiber segment, a fiber Bragg grating [22], a pair of diffraction gratings [23] or a programmable spectral filter [24] imprints a quadratic spectral phase that temporally redistribute and concentrate the energy of the SUT in the central points. Consequently, the resulting signal is sampled at the frequency of the modulation and the peak power at these points is proportional to the initial waveform which is subsequently magnified in a noiseless process [25]. Note that in contrast to our preceding work published in ref. [10] and for which the regime of chromatic dispersion had to be normal, here it can be chosen either anomalous or normal.

The design of our sampling device is rather simple as the final waveform is fully determined by only three parameters: the amplitude $(\theta)$ and frequency $\left(f_{m}\right)$, associated with the temporal sinusoidal phase modulation and the accumulated dispersion $(D)$ linked to the quadratic spectral phase of the second stage. Note that to be fully efficient, the period of the modulation has to be much shorter than the temporal width of the SUT. The sampled waveform $I_{\text {out }}(t)$ can indeed be derived from the SUT $I_{\text {in }}(t)$ (which is here assumed for simplicity to be Fourier transformed) by the following formula :

$$
I_{\text {out }}(t) \simeq \mid F^{-1}\left(\left.F\left(\sqrt{I_{\text {in }}(t)} \exp \left(i \theta \cos \left(2 \pi f_{m} t\right)\right) \exp \left(-i \frac{1}{2} \frac{\lambda^{2}}{2 \pi c} D(2 \pi f)^{2}\right)\right)\right|^{2}\right.
$$

with $c$ being the speed of light, $\lambda$ the central wavelength of the SUT, $t$ and $f$ being the temporal and frequency coordinates respectively. $F$ and $F^{-1}$ stand for the direct and inverse Fourier transform respectively. The magnifying factor $G$ (defined as the power obtained at the 
sampling time, i.e. the peak-power of the resulting ultrashort pulse, divided by the power of the SUT at the same instant before sampling) can be inferred based on the following formula derived in the case of a quasi-continuous wave:

$$
G\left(\theta, D, f_{m}\right) \simeq \max \left(\mid F^{-1}\left(\left.F\left(\exp \left(i \theta \cos \left(2 \pi f_{m} t\right)\right) \quad \exp \left(-i \frac{1}{2} \frac{\lambda^{2}}{2 \pi c} D(2 \pi f)^{2}\right)\right)\right|^{2}\right)\right.
$$

Results of Eq. (2) for $f_{m}$ fixed to $10 \mathrm{GHz}$ are plotted in Fig. 1(c) and show that for each amplitude, the optimum performances (i.e. maximum peak power) are reached for a welldefined dispersion value as predicted by Kokumai in ref. [22] with the approximate formula:

$$
D_{o p t} \simeq \pm \frac{2 \pi c}{\lambda^{2}} \frac{1}{f_{m}^{2}} \frac{1}{30.45 \theta-12.56}
$$

Therefore when modulation amplitude is increased, the best performance is obtained for a lower total dispersion. At the same time, as can be seen in the inset of Fig. 1(c), the higher the modulation amplitude is, the higher the magnifying factor is. 


\section{Experimental setup}

For validating this proposed concept at telecommunication wavelengths, we have implemented the experimental setup depicted in Fig. 2 and made exclusively of commercially available components. In order to generate the nanosecond SUT, an external cavity laser emitting at $1550 \mathrm{~nm}$ is firstly intensity modulated by means of an intensity modulator (IM) that is driven by the electrically filtered output of a pulse pattern generator (PPG) delivering an arbitrary shaped ns signal at a repetition rate of $9.77 \mathrm{MHz}$. Although the performance of this system is independent on the signal power, this wave is amplified up to $10 \mathrm{dBm}$ to avoid a weak signal in the reception stage. Subsequently, the sinusoidal phase is induced in the signal thanks to a phase modulator (PM) driven by a $10 \mathrm{GHz}$ electrical clock that also synchronizes the PPG and the high bandwidth electrical sampling oscilloscope. Compared to our previous work of ref. [10], the use of the PM offers significant advantages in terms of stability and controllability and does not require to involve any high power pulsed wave that has to be polarization multiplexed. The parabolic spectral phase is imprinted on the signal thanks to a liquid-crystal on silicium programmable filter [24] (Finisar Waveshaper device). As the performance of this filter is limited to a total dispersion lower than $80 \mathrm{ps.nm}^{-1}$, we have introduced a $10-\mathrm{km}$ span of single-mode fiber whose dispersion parameter is $-14.5 \mathrm{ps} / \mathrm{nm} / \mathrm{km}$. Consequently, the compensating module achieves a total dispersion $(|\mathrm{D}|)$ close to 145 ps.nm ${ }^{-1}$. Finally, the signal is analyzed in the time domain thanks to an electrical sampling oscilloscope (ESO) with a 70-GHz bandwidth of detection as well as in the optical and electrical spectral domains by means of a high-resolution optical spectrum analyzer (OSA) and a RF spectrum analyzer 


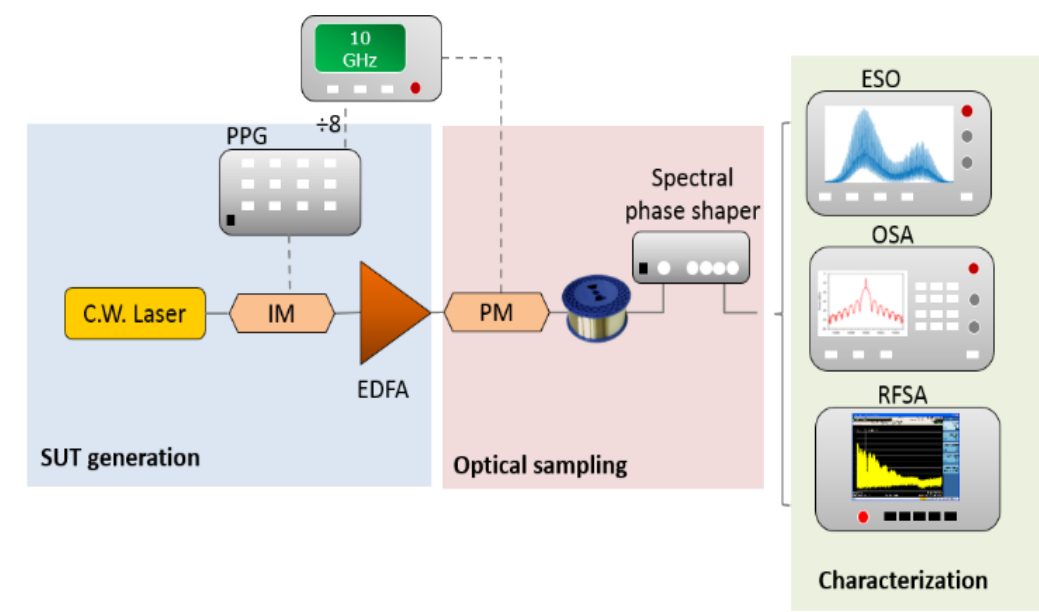

Fig. 2. Experimental setup. IM: intensity modulator, PM: phase modulator, PPG: pulse pattern generator, EDFA: Erbium doped fiber amplifier, ESO: electrical sampling oscilloscope, OSA: optical spectrum analyzer and RFSA: RF spectrum analyzer.

\section{Experimental results}

To fully assess that the present technique enables to sample an arbitrary shaped optical signal without any distortion, we first compare in Fig. 3(a) the normalized input and output intensity profiles obtained for an amplitude of phase modulation of $\theta=0.55 \pi$ and $|\mathrm{D}|$ equals to

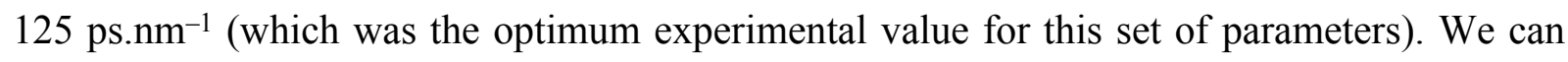
observe that the output waveform (in orange solid line) corresponds to a 10-GHz sampled replica of the initial SUT (blue dotted line) and more specifically, that the details of the signal are accurately reproduced without any distortion independently of the SUT level and complexity. Figure 3(b) displays a close view on only seven temporal periods of the phase 
modulation and confirms the above assumptions. We observe that the full width at half maximum (FWHM) duration of the compressed structures is less than 20 ps. These pulses lie on a residual background that we attribute to the sinusoidal modulation that we employ instead of a perfect parabolic modulation of the temporal phase required for a perfect time-lens. The numerical estimations of Eq. (1) have also been included in the figure in green dashed-line and provide an excellent agreement with our experimental results, thus validating our design rules.

The magnification process can be observed in Fig. 4(a). The intensity profile of the output wave (in orange) corresponds to a temporal intensity comb whose magnitude is exactly proportional to the input signal with an amplitude 3.6 times higher than the one observed when PM is turned off (blue line). In order to check the crucial role played by the quadratic spectral phase, we have recorded the magnifying factor for different values of dispersion. Combining measurements of the spectral shaper alone and the association of the dispersive fiber span with the programmable filter has enabled us to explore the dispersion values $|\mathrm{D}|$ ranging from 0 up to $160 \mathrm{ps} / \mathrm{km} / \mathrm{nm}$. Results are summarized in Fig. 4(b). Following the trends observed in Fig. 1(c), the magnification factor is increasing with the total dispersion until the optimum focusing point is reached. In our experiment a maximum magnifying factor of 3.6 is achieved, which is only $11 \%$ lower than the numerical estimations of Eq. 2 . Note that the experimental results are in quantitative agreement with predictions of Eq. (2) for a modulation amplitude $\theta$ of $0.55 \pi$ (yellow line). 

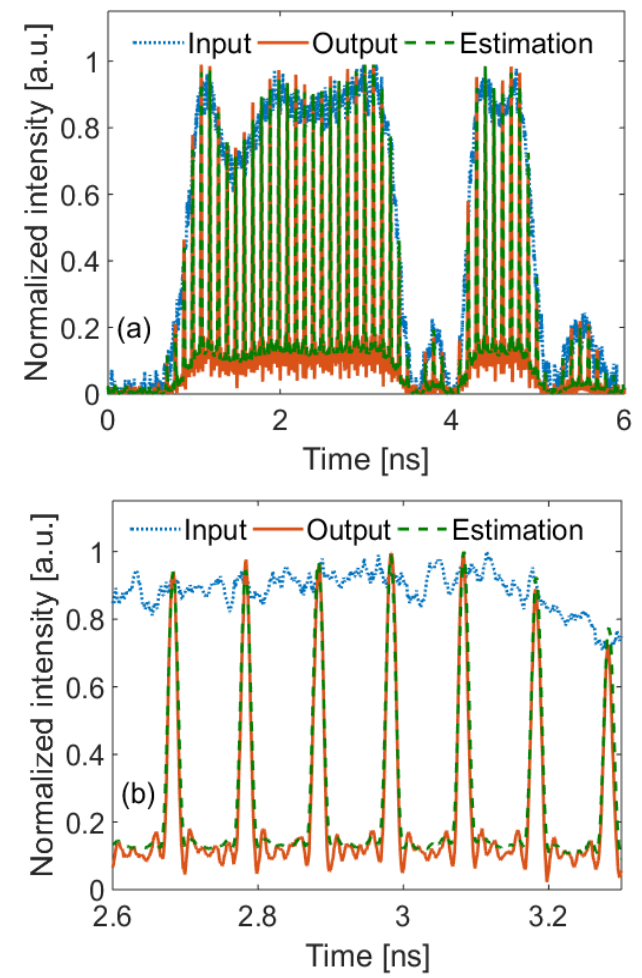

Fig. 3. (a) Normalized intensity profiles of the input (blue dotted line) and output (red line) signals for $\mathrm{D}=-125 \mathrm{ps} \cdot \mathrm{nm}^{-1}$ in the temporal domain. The green dashed line represents the numerical estimation of Eq. (1). The phase amplitude (Vpp/2) is $0.55 \pi$ and $f_{m}=10 \mathrm{GHz}$. (b) Close view of (a). Results are normalized to 1 for their maximum value. 

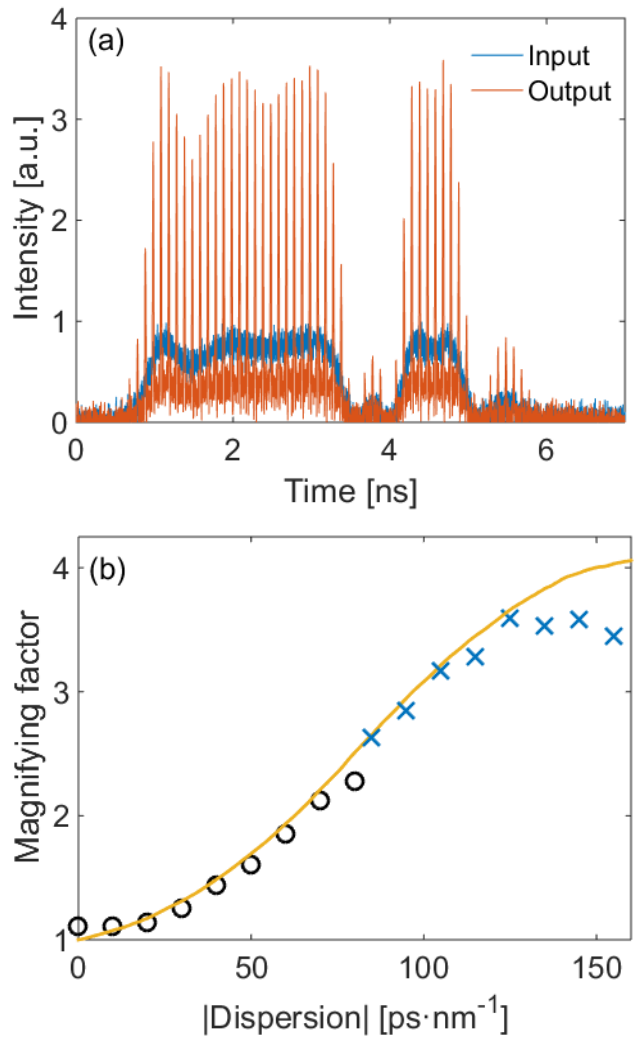

Fig. 4. (a) Intensity profiles of the input (blue dotted line) and output (orange line) signals for $\mathrm{D}=-125 \mathrm{ps} . \mathrm{nm}^{-1}$ in the temporal domain. (b) Experimental magnifying factor as function of the total dispersion of the module obtained with the spectral shaper alone (black circles) and with the combination of dispersive fiber and spectral shaper (blue crosses). Experimental results are compared with the numerical estimation based on Eq. 2 (yellow line).

Finally, the output signal is analyzed in the optical and the RF spectral domains. In the optical domain, the sampling process is characterized by the generation of replicas of the initial waveform regularly spaced by the modulation frequency $(10 \mathrm{GHz})$ as can be seen in Fig. 5(a). The levels of these replica are in excellent agreement with our assumption of a sinusoidal 
phase modulation having a $0.55 \pi \mathrm{rad}$ amplitude and calculated according to Eq. (1) (orange crosses). The optical signal to noise ratio is also excellent, as expected from this noiseless magnification process. Similarly, the initial SUT is reproduced in every $10-\mathrm{GHz}$ harmonics in the RF spectrum with an excellent signal to noise ratio (see Fig. 5(b)).
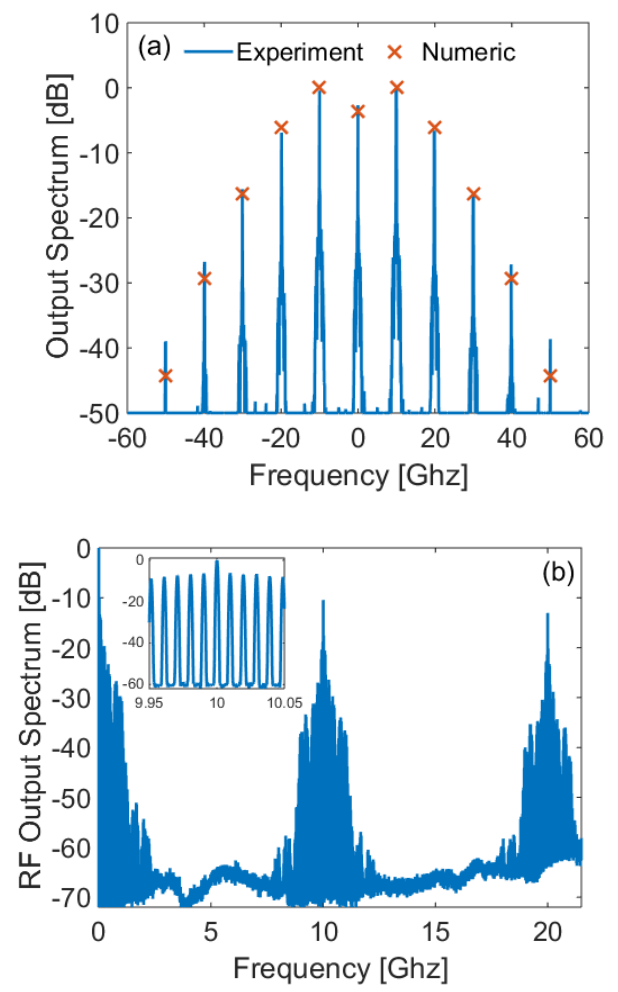

Fig. 5. Spectra of the signal after sampling in the optical domain (a) and in the electrical domain (b) In Fig. 4(a), the maxima of the numerical estimation based on the assumption of a sinusoidal modulation with an amplitude of $0.55 \pi \mathrm{rad}$ are indicated with orange crosses. The inset of Fig. 4(b) represents a close view of the RF spectrum and the spacing between the spectral lines corresponds to the repetition rate of the SUT delivered by the PPG $(9.77 \mathrm{MHz})$. 


\section{Conclusions}

In conclusion, we have suggested and experimentally demonstrated a novel approach to simultaneously sample and magnify the temporal intensity profile of an arbitrary shaped optical signal. Owing to the space/time duality, this technique exploits the temporal analogue of lenticular lenses for which an incoming plane wave is focused at different equally spaced points.

Inspired by a previous nonlinear sampler where Kerr nonlinearity and dispersion act simultaneously and adiabatically in a km-long fiber [10], here we proposed a new linear implementation where tailoring of the temporal and spectral phase profiles are achieved independently by use of compact devices. Moreover, our new implementation has relaxed the need of an external optical high power sampling beam while the use of a programmable spectral shaper offers flexibility to find the optimum operating parameters according to the frequency and amplitude of the temporal phase modulation.

We have experimentally validated our approach by sampling a nanosecond arbitrary shaped signal at a frequency of $10 \mathrm{GHz}$. A magnification factor of 3.6 has been achieved in this proofof-principle experiment. Higher magnifying factor could be reached if the amplitude of the phase modulation is increased up to the levels of a dozen of $\pi$ rad that are already available in the literature. Cascaded phase modulators can also be a solution to improve the results [16], [21]. The aberrations caused by the sinusoidal modulation could be reduced with the current progress of microwave photonic. Finally, let us note that an alternative to the present setup where the second stage induces a parabolic spectral phase could be to use a sharp filter with a central wavelength shifted with respect to incoming signal [26]. The advantage would be to 
generate shorter structures, but at the cost of a severe dissipative process that prevents any magnification. 


\section{Acknowledgments}

We acknowledge fruitful discussions with Hervé Rigneault regarding the space-time analogies. This research is funded by the European Research Council under Grant Agreement 306633, ERC PETAL (www.facebook.com/petal.inside). The authors would like to acknowledge support by the Région Bourgogne (Pari Photcom) and the Agence Nationale de la Recherche (Labex Action ANR-11-LABX-0001-01).

\section{References}

[1] M.A. Duguay, J.W. Hansen, An ultrafast ligth gate, Appl. Phys. Lett. 15 (1969) 192.

[2] T. Kanada, D.L. Franzen, Optical waveform measurement by optical sampling with a mode-locked laser diode, Opt. Lett. 11 (1986) 4-6.

[3] N. Yamada, S. Nogiwa, H. Ohta, 640-Gb/s OTDM signal measurement with highresolution optical sampling system using wavelength-tunable soliton pulses, IEEE Photonics Technol. Lett. 16 (2004) 1125-1127.

[4] J. Li, J. Hansryd, P.O. Hedekvist, P.A. Andrekson, S.N. Knudsen, 300-Gb/s eyediagram measurement by optical sampling using fiber-based parametric amplification, IEEE Photonics Technol. Lett. 13 (2001), 987-989.

[5] S. I. Oda, A. Maruta, K. Kitayama, All-optical quantization scheme based on fiber nonlinearity, IEEE Photon.Technol. Lett., 16 (2004) 587-589.

[6] J. Li, M. Westlund, H. Sunnerud, B.E. Olsson, M. Karlsson, P.A. Andrekson, 0.5-Tb/s eye-diagram measurement by optical sampling using XPM-induced wavelength shifting in highly nonlinear fiber, IEEE Photonics Technol. Lett. 16 (2004) 566-568. 
[7] P.A. Andrekson, M. Westlund, Nonlinear optical fiber based high resolution all-optical waveform sampling, Laser Photon. Rev. 1 (2007) 231-248.

[8] S. Zhang, Y. Liu, Y. Liu, H. J.S. Dorren, All-optical sampling exploiting nonlinear polarization rotation in a single semiconductor optical amplifier, Opt. Commun. 285 (2012) 1001-1004.

[9] C. Xu, X. Liu, Photonic analog-to-digital converter using soliton self-frequency shift and interleaving spectral filters, Opt. Lett. 28 (2003) 986-988.

[10] J. Nuño, M. Gilles, M. Guasoni, C. Finot, J. Fatome, All-optical sampling and magnification based on XPM-induced focusing, Opt. Express 24 (2016) 24921-24929.

[11] J. Nuño, M Gilles, M Guasoni, B Kibler, C Finot, J Fatome, 40 GHz pulse source based on cross-phase modulation-induced focusing in normally dispersive optical fibers, Optics letters 41 (2016) 1110-1113.

[12] B. H. Kolner, Space-time duality and the theory of temporal imaging, IEEE J. Quantum Electron. 30 (1994) 1951-1963.

[13] J. Van Howe, C. Xu, Ultrafast Optical Signal Processing Based Upon Space-Time Dualities, J. Lightw. Technol. 24 (2006) 2649-2662.

[14] J. A. Giordmaine, M. A. Duguay, J. W. Hansen, Compression of optical pulses, IEEE J. Quantum Electron., QE-4 (1968) 252-255.

[15] B. H. Kolner, M. Nazarathy, Temporal imaging with a time lens, Opt. Lett. 14 (1989) 630-632.

[16] A. A. Godil, B. A. Auld, D. M. Bloom, Picosecond time-lenses, IEEE J. Quantum Electron. 30 (1994) 827-837.

[17] D. Grischkowsky, A. C. Balant, Optical pulse compression based on enhanced frequency chirping Appl. Phys. Lett. 41 (1982) 1-3. 
[18] W. J. Tomlinson, R. H. Stolen, C. V. Shank, Compression of optical pulses chirped by self-phase modulation in fibers, J. Opt. Soc. Am. B. 1 (1984) 139-149.

[19] Q. Z. Wang, P. P. Ho, and R. R. Alfano, Degenerate cross-phase modulation for pulse compression and amplification of ultrashort laser pulses, Optics Letters, 15 (1990) 1023-1025.

[20] D. Grischkowsky, Optical Pulse Compression, Appl. Phys. Lett. 25 (1974) 566-568.

[21] T. Yamamoto, T. Komukai, K. Suzuki, A. Takada, High-reflectivity-resolution coherent optical frequency domain reflectometry using optical frequency comb source and tunable delay line, J. Lightw. Technol. 27 (2009) 4297-4305.

[22] T. Komukai, T. Yamamoto, S. Kawanishi, Optical pulse generator using phase modulator and linearly chirped fiber Bragg gratings, IEEE Photon. Technol. Lett., 17 (2005) $1746-1748$.

[23] T. Kobayashi, H. Yao, K. Amano, Y. Fukushima, A. Morimoto, T. Sueta, Optical pulse compression using high-frequency electrooptic phase modulation, IEEE J. Quantum Electron., 24 (1988) 382-387.

[24] M. A. F. Roelens, S. Frisken, J. A. Bolger, D. Abakoumov, G. Baxter, S. Poole, B. J. Eggleton, Dispersion trimming in a reconfigurable wavelength selective switch, J. Lightw. Technol. 26 (2008) 73-78.

[25] R. Maram, J. Van Howe, M. Li, J. Azaña, Noiseless intensity amplification of repetitive signals by coherent addition using the temporal Talbot effect, Nat. Commun. 5 (2014) 5163.

[26] B. H. Chapman, A. V. Doronkin, S. V. Popov, J. R. Taylor, All-fiber integrated 10 $\mathrm{GHz}$ repetition rate femtosecond laser source based on Raman compression of pulses generated through spectral masking of a phase-modulated diode, Opt. Lett. 37 (2012) 30993101. 